\title{
Benign Infantile Epilepsy Mimicking Reflex Anoxic Seizures in an Infant with PRRT2 Gene Mutation
}

\author{
Victoria Vlachou $^{1}$ (D) Vivian Chu $^{1} \cdot$ Efterpi Pavlidou $^{2} \cdot$ Naila Ismayilova $^{1} \cdot$ Mankad Kshitij $^{3} \cdot$ Maria Kinali $^{1}$
}

Received: 4 September 2017 / Accepted: 27 November 2017 / Published online: 18 December 2017

(C) Dr. K C Chaudhuri Foundation 2017

To the Editor: A 12-mo-old Caucasian girl presented with recurrent seizures since the age of nine months. She was the first child of non-consanguineous parents. She would become vacant with facial pallor, occasional lips cyanosis and isolated upper limb jerky movement. A tonic stiffening of upper limbs, myoclonic movements of fingers and facial musculature contraction would follow after five minutes. She would then fall asleep for two hours. The triggers were difficult to identify, but speculated to include frustration, fright or a feeling of abandonment.

Neurological examination was normal. Routine, sleepdeprived and 24-h electroencephalography (EEG) telemetry study were all reported as normal. Brain MRI did not reveal any pathological findings and cardiac investigations were also normal. Given the unusual presentation, a genetic cause was suspected. Targeted sequence analysis of the PRRT2 gene, the leading cause for paroxysmal disorders, detected the exon 2 c.649dupC p.(Arg217Profs*8) PRRT2 variant in the heterozygous state. The father was found to share the same mutation.

PRRT2 encodes for the proline-rich transmembrane protein 2 in the developing nervous system and interacts with synaptosomal associated protein 25 (SNAP-25), involved in the interaction of $\mathrm{Ca} 2+-$ triggered release of neurotransmitters at the presynaptic terminals [1]. To date, some 1500 patients with approximately seventy different $P R R T 2$ mutations have been reported. Of these, 5.5\% (79/1444) occurred de novo, while $87.1 \%$ (1258/1444) are familial in origin [2]. PRRT2 mutations have been mainly associated with Benign Familial

Maria Kinali

m.kinali@imperial.ac.uk

1 Department of Pediatric Neurology, Chelsea and Westminster Hospital, NHS Foundation Trust, Imperial College University, 369 Fulham Road, London SW10 9NH, UK

2 Department of Pediatrics, University Hospital of Thessaloniki, AHEPA, Thessaloniki, Greece

3 Department of Pediatric Radiology, Chelsea and Westminste Hospital, NHS Foundation Trust, Imperial College University, London, UK
Infantile Epilepsy, Paroxysmal Kinesigenic Dyskinesia and Infantile Convulsions and Choreoathetosis [2] and lately with benign myoclonus of early infancy [3] and paroxysmal hypnogenic dyskinesia (PHD) [4].

At $25 \mathrm{mo}$ of age, the child has been seizure free with a normal psychomotor development and no anticonvulsant medications. Though her presentation was highly evocative of reflex anoxic seizures, her clinical phenotype and the PRRT2 loss of function mutation corroborated the diagnosis of a benign form of epilepsy, according to ILAE classification of 2010 [5]. Currently, PRRT2 physiological function remains unclear, even though a marked pleiotropy and variable penetrance of its mutations are well-described. Our case herein concurs in expanding the clinical spectrum of PRRT2 related disorders.

Acknowledgements The authors would like to thank the family of the patient for giving them permission for publication.

\section{Compliance with Ethical Standards}

Conflict of Interest None.

Source of Funding None.

\section{References}

1. Heron SE, Dibbens LM. Role of PRRT2 in common paroxysmal neurological disorders: a gene with remarkable pleiotropy. J Med Genet. 2013;50:133-9.

2. Ebrahimi-Fakhari D, Saffari A, Westenberger A, Klein C. The evolving spectrum of PRRT2-associated paroxysmal disease. Brain. 2015:138:3476-95.

3. Maini I, Iodice A, Spagnoli C, et al. Expanding phenotype of PRRT2 gene mutations: a new case with epilepsy and benign myoclonus of early infancy. Eur J Paediatr Neurol. 2016;20:454-6.

4. Liu XR, Huang D, Wang J, et al. Paroxysmal hypnogenic dyskinesia is associated with mutations in the PRRT2 gene. Neurol Genet. 2016;2:e66.

5. Berg AT, Berkovic SF, Brodie MJ, et al. Revised terminology and concepts for organization of seizures and epilepsies: report of the ILAE commission on classification and terminology, 2005-2009. Epilepsia. 2010;51:676-85. 